\title{
May 2016 CPD question 2
}

CPD question 2 in the May 2015 SAMJ (Vol. 106, No. 5) should have read as follows: 'Women who die from bleeding associated with CD would be expected to have lost more than $2500 \mathrm{~mL}$ of blood.' The online version of the CPD (http://dx.doi.org/10.7196/SAMJ.2016.v106i5.10929) was corrected on 8 June 2016.

SAfr Med J 2016;106(7):737. DOI:10.7196/SAMJ.2016.v106i7.11157 